\title{
Dark matter: A spin one half fermion field with mass dimension one?
}

\author{
D. V. Ahluwalia-Khalilova ${ }^{*}$ and D. Grumiller ${ }^{\oplus}$ \\ a ASGBG/CIU, Department of Mathematics, Ap. Postal C-600, University of Zacatecas, ZAC 98060, Mexico \\ ${ }^{b}$ Institut für Theoretische Physik, Universität Leipzig, Augustusplatz 10, D-04109 Leipzig, Germany
}

(Dated: October 15, 2004)

\begin{abstract}
We report an unexpected theoretical discovery of a spin one half matter field with mass dimension one. It is based on a complete set of eigenspinors of the charge conjugation operator. Due to its unusual properties with respect to charge conjugation and parity it belongs to a non standard Wigner class. Consequently, the theory exhibits non-locality with $(C P T)^{2}=-\mathbb{I}$. Its dominant interaction with known forms of matter is via Higgs, and with gravity. This aspect leads us to contemplate it as a first-principle candidate for dark matter.

PACS numbers: 11.10.Lm 11.30.Cp 11.30.Er 95.35.+d
\end{abstract}

\section{INTRODUCTION}

The twentieth century may be described accurately as an era of local quantum field theories (QFTs). The concepts emerged in its first half, after unifying quantum mechanics, special relativity, and classical field theory. The applications were found and studied in detail, especially in the second half. This has culminated in the fantastically successful Standard Model (SM) of particle physics which describes all known forces of Nature except for gravity [1]. As the unification of gravity with the quantum realm is still a work in progress it is worthwhile to tread gingerly. A safe, if somewhat vague, statement is that quantum gravity induces non-locality. This is realized in different ways explicitly in string theory [2], in loop quantum gravity [3] , and in non-commutative field theories [4], to name just the most prominent candidates for quantum gravity. Clearly, abandoning locality is a big step. Therefore, we would like to be as conservative as possible regarding further deviations from the SM and its foundation in local QFT.

Non standard Wigner classes. - Just dropping the postulate of locality is not specific enough. The path we will take is built upon the classic framework of Wigner 5] where particles are described by irreducible projective representations of the full Poincaré group. At the kinematic level, they are labeled by its Casimir invariants. In addition, they are endowed with certain behavior under parity $P$ and charge conjugation $C$ as distinguished by various Wigner classes. This notion of particles is a corner stone of any description of the low energy regime that we are able to explore experimentally ("low" with respect to the Planck scale). Thus, we would like to keep it, and advocate an ab initio exploration of a non standard Wigner class (NSWC). At this point two important facts are recalled: (a) For the standard Wigner classes, the $P$ and $C$ anticommute for fermions and commute for bosons; this is true for all particles of the SM;

*Electronic address: dva-k@heritage.reduaz.mx

${ }^{\dagger}$ Electronic address: grumiller@itp.uni-leipzig.de and, (b) Any non-trivial theory built upon a NSWC has to be non-local [ 6 ]. The second property is the reason why the NSWCs are discarded normally. However, we regard it not as a disadvantage but as a virtue, because non-locality is introduced in a definite way with no free parameters apart from particle properties. For the sake of concreteness we shall focus on spin one half.

Neutrality. - The concrete path we take is guided by the following observation. Typically, what localizes the otherwise extended field configurations like solitons is a conserved (topological) charge [13. In the absence of such charges there is nothing that protects the field from spreading; and therefore, in such situations, the emergence of non-locality may not be completely surprising. We are thus led to consider Eigenspinors of the charge conjugation operator; abbreviated as Elko from the German "Eigenspinoren des Ladungskonjugationsoperators". As we will show below, the assumption of neutrality alone will not only lead to a special type of non-locality with a certain mass dependence, but also to a NSWC with $[C, P]=0$. Thus, we need solely one postulate, namely that the new matter field we seek to describe is built upon Elko.

The discovery. - The constructed matter field, despite carrying spin one half, is endowed with mass dimension one. This aspect, as we will argue, makes it a firstprinciple dark matter candidate.

Preliminaries. - The derivation of the Dirac equation as presented, e.g. in Ref. 7, carries a quantum mechanical aspect in allowing for the fact that the two Weyl spaces may carry a relative phase; and concurrently a relativistic element via the Lorentz transformation properties of the Weyl spinors. In turn, the very existence of the latter depends on the existence of two spacetime $S U(2)$ s, with the following generators of transformation: $\mathbf{A}_{ \pm}=\frac{1}{2}(\mathbf{J} \mp i \mathbf{K})$. The $\mathbf{J}$ and $\mathbf{K}$ represent the generators of rotations and boosts, respectively. We use the Pauli matrices $\boldsymbol{\sigma}=\left(\sigma_{1}, \sigma_{2}, \sigma_{3}\right)$ and the Dirac matrices $\gamma^{\mu}$ in standard Weyl representation, subsequently. For $\mathbf{J}=\boldsymbol{\sigma} / 2$ and $\mathbf{A}_{+}=\mathbf{0}\left[\mathbf{A}_{-}=\mathbf{0}\right]$ we have the $(1 / 2,0)$ right-handed $[(0,1 / 2)$ left-handed] Weyl space where $\mathbf{K}$ equals $-i \boldsymbol{\sigma} / 2[+i \boldsymbol{\sigma} / 2]$. From the womb of this structure emerges the Dirac equation, $\left(\gamma^{\mu} p_{\mu} \pm m \mathbb{I}\right) \psi(\mathbf{p})=0$, 
which carries the particle-antiparticle symmetry via the operation of charge conjugation. In Weyl realization, the operator associated with it is

$$
C=\left(\begin{array}{cc}
\mathbb{O} & i \Theta \\
-i \Theta & \mathbb{O}
\end{array}\right) K
$$

where $K$ complex conjugates a spinor appearing on its right and $\Theta$ is Wigner's spin half time reversal operator. We employ the representation $\Theta=-i \sigma_{2}$. Note that $\Theta[\boldsymbol{\sigma} / 2] \Theta^{-1}=-[\boldsymbol{\sigma} / 2]^{*}$. Equation (10) yields the expected $C=-\gamma^{2} K$. The boost operator, $\kappa^{+} \oplus \kappa^{-}$, with

$$
\kappa^{ \pm}=\exp \left( \pm \frac{\boldsymbol{\sigma}}{2} \cdot \boldsymbol{\varphi}\right)=\sqrt{\frac{E+m}{2 m}}\left(\mathbb{I} \pm \frac{\boldsymbol{\sigma} \cdot \mathbf{p}}{E+m}\right),
$$

and the $(1 / 2,0) \oplus(0,1 / 2)$-space charge conjugation operator, $C$, commute. In terms of energy $E$ and momentum $\mathbf{p}=p \widehat{\mathbf{p}}$ the boost parameter, $\boldsymbol{\varphi}=\varphi \widehat{\mathbf{p}}$, is defined as $\cosh (\varphi)=E / m, \sinh (\varphi)=p / m$, where $m$ is the mass.

\section{FORMAL STRUCTURE OF ELKO}

We have summarized above the origin and form of $C$. We now proceed to obtain its eigenspinors. If $\phi_{L}(\mathbf{p})$ transforms as a left handed spinor, then $(\zeta \Theta) \phi_{L}^{*}(\mathbf{p})$ transforms as a right handed spinor - where $\zeta$ is an unspecified phase. As a consequence, the following spinors belong to the $\left(\frac{1}{2}, 0\right) \oplus\left(0, \frac{1}{2}\right)$ representation space ${ }^{1}$ :

$$
\lambda(\mathbf{p})=\left(\begin{array}{c}
(\zeta \Theta) \phi_{L}^{*}(\mathbf{p}) \\
\phi_{L}(\mathbf{p})
\end{array}\right)
$$

These become Eigenspinors of $C$, viz., Elko, with real eigenvalues if the phase $\zeta$ is restricted to $\zeta= \pm i$ :

$$
C \lambda(\mathbf{p})= \pm \lambda(\mathbf{p})
$$

The plus [minus] sign yields self-conjugate [anti selfconjugate] spinors: $\lambda^{S}(\mathbf{p})\left[\lambda^{A}(\mathbf{p})\right]$.

Explicit form of Elko. - To obtain explicit expressions for $\lambda(\mathbf{p})$ we consider the rest frame $(\mathbf{p}=\mathbf{0})$ and decompose the $\phi_{L}(\mathbf{0})$ into helicity eigenstates: $\boldsymbol{\sigma} \cdot \widehat{\mathbf{p}} \phi_{L}^{ \pm}(\mathbf{0})=$ $\pm \phi_{L}^{ \pm}(\mathbf{0})$. Taking $\widehat{\mathbf{p}}=(\sin \theta \cos \phi, \sin \theta \sin \phi, \cos \theta)$, yields

$$
\begin{aligned}
& \phi_{L}^{+}(\mathbf{0})=\sqrt{m} e^{i \vartheta_{1}}\left(\begin{array}{c}
\cos (\theta / 2) e^{-i \phi / 2} \\
\sin (\theta / 2) e^{i \phi / 2}
\end{array}\right), \\
& \phi_{L}^{-}(\mathbf{0})=\sqrt{m} e^{i \vartheta_{2}}\left(\begin{array}{c}
\sin (\theta / 2) e^{-i \phi / 2} \\
-\cos (\theta / 2) e^{i \phi / 2}
\end{array}\right) .
\end{aligned}
$$

\footnotetext{
1 There is a second set of spinors that may be built by starting with a right-handed Weyl spinor $\phi_{R}(\mathbf{p})$, and the observation that $(\zeta \Theta)^{*} \phi_{R}^{*}(\mathbf{p})$ transforms as a left-handed Weyl spinor. Due to its equivalence with the set considered in the present work we postpone its details to [8].
}

We set $\vartheta_{1}=\vartheta_{2}=0^{2}$. This leads to four spinors

$$
\lambda_{\{\mp, \pm\}}(\mathbf{0})=\left(\begin{array}{c}
\zeta \Theta\left[\phi_{L}^{ \pm}(\mathbf{0})\right]^{*} \\
\phi_{L}^{ \pm}(\mathbf{0})
\end{array}\right) .
$$

Two of these are [anti] self conjugate and arise from setting $\zeta=+i[\zeta=-i]$. These are denoted by $\lambda_{\{\mp, \pm\}}^{S}(\mathbf{0})$ $\left[\lambda_{\{\mp, \pm\}}^{A}(\mathbf{0})\right]$. The first [second] helicity entry refers to the $\left(\frac{1}{2}, 0\right)\left[\left(0, \frac{1}{2}\right)\right]$ transforming component of the $\lambda(\mathbf{p})$. Equations (2) and (6) yield the boosted spinors:

$$
\lambda_{\{\mp, \pm\}}^{S / A}(\mathbf{p})=\sqrt{\frac{E+m}{2 m}}\left(1 \mp \frac{p}{E+m}\right) \lambda_{\{\mp, \pm\}}^{S / A}(\mathbf{0})
$$

In the massless limit $\lambda_{\{-,+\}}^{S / A}(\mathbf{p})$ identically vanishes while $\lambda_{\{+,-\}}^{S / A}(\mathbf{p})$ does not. Moreover, the relation, $\boldsymbol{\sigma}$. $\widehat{\mathbf{p}} \Theta\left[\phi_{L}^{ \pm}(\mathbf{0})\right]^{*}=\mp \Theta\left[\phi_{L}^{ \pm}(\mathbf{0})\right]^{*}$, physically implies the following: $\Theta\left[\phi_{L}^{ \pm}(\mathbf{0})\right]^{*}$ has opposite helicity of $\phi_{L}^{ \pm}(\mathbf{0})$. Since $\boldsymbol{\sigma} \cdot \widehat{\mathbf{p}}$ commutes with $\kappa^{ \pm}$this result holds for all $\mathbf{p}$. We thus have the important property for Elko: they are not single helicity objects. That is, Elko cannot be eigenspinors of the helicity operator. The same shall be assumed for one-particle states.

A new dual for Elko. - For any $\left(\frac{1}{2}, 0\right) \oplus\left(0, \frac{1}{2}\right)$ spinor $\xi(\mathbf{p})$, the Dirac dual spinor $\bar{\xi}(\mathbf{p})$ is defined as $\bar{\xi}(\mathbf{p}):=$ $\xi^{\dagger}(\mathbf{p}) \gamma^{0}$. It is readily verified that with respect to the Dirac dual, the Elko have an imaginary bi-orthogonal norm, which is a hindrance to physical interpretation and quantization. Therefore, we define a new dual which is required to have the property that: (a) It yields an invariant real definite norm, and (b) It must secure a positive definite norm for two of the four Elko's, and negative definite norm for the remaining two. Up to an irrelevant relative sign, a unique definition, which we call Elko dual, is

$$
\lambda_{\{\mp, \pm\}}^{S / A}(\mathbf{p}):= \pm i\left[\lambda_{\{ \pm, \mp\}}^{S / A}(\mathbf{p})\right]^{\dagger} \gamma^{0} .
$$

With the Elko dual thus defined, we now have, by construction, the orthonormality relations

$$
\begin{aligned}
& \vec{\lambda}_{\alpha}^{S}(\mathbf{p}) \lambda_{\alpha^{\prime}}^{I}(\mathbf{p})=+2 m \delta_{\alpha \alpha^{\prime}} \delta_{S I}, \\
& \vec{\lambda}_{\alpha}^{A}(\mathbf{p}) \lambda_{\alpha^{\prime}}^{I}(\mathbf{p})=-2 m \delta_{\alpha \alpha^{\prime}} \delta_{A I},
\end{aligned}
$$

where $I \in\{S, A\}$; and the completeness relation

$$
\frac{1}{2 m} \sum_{\alpha}\left[\lambda_{\alpha}^{S}(\mathbf{p}) \vec{\lambda}_{\alpha}^{S}(\mathbf{p})-\lambda_{\alpha}^{A}(\mathbf{p}) \vec{\lambda}_{\alpha}^{A}(\mathbf{p})\right]=\mathbb{I},
$$

which clearly shows the necessity of the anti selfconjugate spinors. In the above equations, the subscript

\footnotetext{
2 This choice is important for the specific norms given in Eqs. 9a and $9 \mathrm{~b}$.
} 
$\alpha$ ranges over two possibilities: $\{+,-\},\{-,+\}$. The detailed structure underlying the completeness relation resides in the following spin sums

$$
\begin{aligned}
& \sum_{\alpha} \lambda_{\alpha}^{S}(\mathbf{p}) \vec{\lambda}_{\alpha}^{S}(\mathbf{p})=+m[\mathbb{I}+\mathcal{G}(\mathbf{p})] \\
& \sum_{\alpha} \lambda_{\alpha}^{A}(\mathbf{p}) \vec{\lambda}_{\alpha}^{A}(\mathbf{p})=-m[\mathbb{I}-\mathcal{G}(\mathbf{p})] ;
\end{aligned}
$$

which together define $\mathcal{G}(\mathbf{p})$. A detailed calculation shows that $\mathcal{G}$ is an odd function of $\mathbf{p}$ :

$$
\mathcal{G}(\mathbf{p})=-\mathcal{G}(-\mathbf{p})
$$

a result which carries considerable significance for the discussion following Eq. (19). Equations (9a)-(11b) have their direct counterparts in Dirac's construct.

Behavior under $C, P$ and T. - It appears to be standard textbook wisdom that for bosons [fermions] particle and antiparticle have same [opposite] relative intrinsic parity. To our knowledge the only textbook which tells a more intricate story is that by Weinberg [1]. The only known explicit construct of a theory which challenges the conventional wisdom was reported about a decade ago [9]. In that pure spin one bosonic theory particles and antiparticles carry opposite, rather than same, relative intrinsic parity. It manifests itself through anticommutativity, as opposed to commutativity, of the $(1,0) \oplus(0,1)$ space's $C$ and $P$ operators. In a somewhat parallel fashion we shall now show that for the spin half Elko $C$ and $P$ commute, rather than anticommute as they do for the Dirac case. The $P$ acting on Elko yields

$$
P \lambda_{\{\mp, \pm\}}^{S}(\mathbf{p})= \pm i \lambda_{\{ \pm, \mp\}}^{A}(\mathbf{p}),
$$

and the same equation with a minus sign on the l.h.s. for $\lambda^{A} \leftrightarrow \lambda^{S}$. That is, Elko are not eigenspinors of the parity operator. Applying it twice establishes $P^{2}=-\mathbb{I}$; as opposed to Dirac spinors where $P^{2}=+\mathbb{I}$. Under time reversal $T=i \gamma^{5} C$ we obtain

$$
T \lambda_{\alpha}^{S}(\mathbf{p})=-i \lambda_{\alpha}^{A}(\mathbf{p}), \quad T \lambda_{\alpha}^{A}(\mathbf{p})=+i \lambda_{\alpha}^{S}(\mathbf{p}),
$$

implying $T^{2}=-\mathbb{I}$. It is now a simple exercise to show

$$
\text { ELko : } \quad[C, P]=0, \quad[C, T]=0, \quad\{P, T\}=0 .
$$

This proves our claim that Elko belong to a NSWC [5]. We confirm also Wigner's expectation $(C P T)^{2}=-\mathbb{I}$ and reconcile with Weinberg's observation (appendix C of chapter 2 in [1]) due to Elko's dual helicity nature.

\section{PHYSICAL PROPERTIES OF ELKO}

A spin one half matter field with mass dimension one.- An Elko-based quantum field with well-defined
$C P T$ properties may now be introduced

$$
\begin{array}{r}
\eta(x)=\int \frac{d^{3} p}{(2 \pi)^{3}} \frac{1}{\sqrt{2 m E(\mathbf{p})}} \sum_{\beta}\left[c_{\beta}(\mathbf{p}) \lambda_{\beta}^{S}(\mathbf{p}) \mathrm{e}^{-i p_{\mu} x^{\mu}}\right. \\
\left.+c_{\beta}^{\dagger}(\mathbf{p}) \lambda_{\beta}^{A}(\mathbf{p}) \mathrm{e}^{+i p_{\mu} x^{\mu}}\right],
\end{array}
$$

with the expected anti-commutation relations

$$
\begin{aligned}
& \left\{c_{\beta}(\mathbf{p}), c_{\beta^{\prime}}^{\dagger}\left(\mathbf{p}^{\prime}\right)\right\}=(2 \pi)^{3} \delta^{3}\left(\mathbf{p}-\mathbf{p}^{\prime}\right) \delta_{\beta \beta^{\prime}} \\
& \left\{c_{\beta}^{\dagger}(\mathbf{p}), c_{\beta^{\prime}}^{\dagger}\left(\mathbf{p}^{\prime}\right)\right\}=\left\{c_{\beta}(\mathbf{p}), c_{\beta^{\prime}}\left(\mathbf{p}^{\prime}\right)\right\}=0
\end{aligned}
$$

for the creation and annihilation operators $c_{\beta}^{\dagger}(\mathbf{p})$ and $c_{\beta}(\mathbf{p})$, respectively. Its Elko dual $\vec{\eta}(x)$ is obtained by replacing everywhere $\lambda(\mathbf{p})$ with its Elko dual, exchanging $c$ with $c^{\dagger}$, and swapping $i p_{\mu} x^{\mu} \leftrightarrow-i p_{\mu} x^{\mu}$. The propagator follows from textbook methods. It entails evaluation of $\left\langle\left|T\left(\eta\left(x^{\prime}\right) \vec{\eta}(x)\right)\right|\right\rangle$, where $T$ is the fermionic timeordering operator, and |\rangle is the vacuum state. The result in terms of the spin sums reads

$$
\begin{aligned}
\mathcal{S}\left(x-x^{\prime}\right) & =-\int \frac{d^{3} p}{(2 \pi)^{3}} \frac{i}{2 m E(\mathbf{p})} \\
\times & \sum_{\beta}\left[\theta\left(t^{\prime}-t\right) \lambda_{\beta}^{S}(\mathbf{p}) \vec{\lambda}_{\beta}^{S}(\mathbf{p}) \mathrm{e}^{-i p_{\mu}\left(x^{\prime \mu}-x^{\mu}\right)}\right. \\
& \left.-\theta\left(t-t^{\prime}\right) \lambda_{\beta}^{A}(\mathbf{p}) \vec{\lambda}_{\beta}^{A}(\mathbf{p}) \mathrm{e}^{+i p_{\mu}\left(x^{\prime \mu}-x^{\mu}\right)}\right] .
\end{aligned}
$$

On using Eqs. (11a) and 11b for the spins sums it simplifies to

$$
\mathcal{S}\left(x-x^{\prime}\right)=\int \frac{d^{4} p}{(2 \pi)^{4}} \mathrm{e}^{i p_{\mu}\left(x^{\mu}-x^{\prime \mu}\right)} \frac{\mathbb{I}+\mathcal{G}(\mathbf{p})}{p_{\mu} p^{\mu}-m^{2}+i \epsilon} .
$$

In (19), the limit $\epsilon \rightarrow 0^{+}$is understood. The structure of the obtained propagator differs from that of Dirac because in this latter case $(\mathbb{I} \pm \mathcal{G}(\mathbf{p}))$ appearing in the spin sums is replaced by its counterpart - $\left(\mathbb{I} \pm \gamma_{\mu} p^{\mu} / m\right)$ (with plus sign giving the spin sum for particle spinors $u_{h}(\mathbf{p})$, while the minus sign yielding the same for antiparticle spinors $\left.v_{h}(\mathbf{p})\right)$. Exploiting the property (12), it is clear that in the absence of a preferred direction, such as the one arising from a fixed background, like a reference fluid, a thermal bath or an external magnetic field, to name just a few, the second term in Eq. (19) identically vanishes; and as a result, Eq. (19) reduces to the Klein-Gordon propagator. Consequently, the field $\eta(x)$ carries mass dimension one. It forbids particles described by the theory to enter $S U(2)_{L}$ doublets of the SM. The field $\eta(x)$ thus becomes a first-principle candidate for dark matter as will be discussed below in more detail.

The identity ${ }^{3}\left(\gamma_{\mu} p^{\mu} \delta_{\alpha}^{\beta} \pm i m \mathbb{I} \varepsilon_{\alpha}^{\beta}\right) \lambda_{\beta}^{S / A}(\mathbf{p})=0$, follows as a simple algebraic exercise of applying $\gamma_{\mu} p^{\mu}$ to $\lambda^{S / A}(\mathbf{p})$

\footnotetext{
${ }^{3}$ Here $\delta_{\beta}^{\alpha}$ is the usual Kronecker delta, the antisymmetric symbol is defined as $\varepsilon_{\{+,-\}}^{\{-,+\}}:=-1$, and $+(-)$ sign is to be taken for self-conjugate (anti self-conjugate) spinors.
} 
[8, 10]. It cannot be interpreted as Dirac equation with an off-diagonal mass term. Instead, the mentioned identity shows that Elko satisfy the Klein-Gordon equation, $\left(p_{\mu} p^{\mu}-m^{2}\right) \lambda^{S / A}(\mathbf{p})=0$.

As a further consistency check, from the Lagrangian density

$$
\left.\mathcal{L}^{\text {free }}=\partial^{\mu}\right\urcorner(x) \partial_{\mu} \eta(x)-m^{2} \vec{\eta}(x) \eta(x),
$$

one may construct the Hamiltonian density and it turns out that the anti-commutation relations (16), (17) are compatible with positive energy, like in the Dirac case.

Non-locality. - Given (15), its dual $\vec{\eta}(x)$, as well as the canonical momentum $\pi(x)$ implied by Eq. (20), as input it is easy to calculate the field anticommutators. We find that $\left\{\eta(\mathbf{x}, t), \vec{\eta}\left(\mathbf{x}^{\prime}, t\right)\right\}$ vanishes while $\left\{\eta(\mathbf{x}, t), \pi\left(\mathbf{x}^{\prime}, t\right)\right\}=i \delta^{3}\left(\mathbf{x}-\mathbf{x}^{\prime}\right)$. This is as expected on the basis of a local QFT. The departure from locality is contained in the result that $\left\{\eta(\mathbf{x}, t), \eta\left(\mathbf{x}^{\prime}, t\right)\right\}$ and $\left\{\pi(\mathbf{x}, t), \pi\left(\mathbf{x}^{\prime}, t\right)\right\}$ do not vanish. The emergent nonlocality is captured by the expression

$$
\frac{\mathrm{d}}{\mathrm{d} m}\left[m \int_{\mathbf{X}-\mathbf{X}^{\prime}}\left\langle\left|\left\{\eta(\mathbf{x}, t), \eta\left(\mathbf{x}^{\prime}, t\right)\right\}\right|\right\rangle\right]=\frac{1}{m} \gamma^{1} \gamma^{0}
$$

In the limit of large $m$ non-locality becomes negligible. It is worth emphasizing that non-locality for Elko emerges as a higher order effect; for it resides entirely in those expectation values where two Elko fields, or two momenta, appear together.

Elko as dark matter candidate.- Having established non-locality, CPT-properties and mass dimension one, the physics of Elko becomes even more interesting when coupling to the matter content of the SM is considered. Since interaction terms with mass dimension greater than four will be assumed to be suppressed by some fundamental mass scale, say, the Planck scale, focus will be solely on power counting renormalizable and super-renormalizable terms ${ }^{4}$. It is easy to check that none of the latter are present: for a scalar interaction term Elko must appear in even powers, so superrenormalizable terms must contain exactly two Elkos and one other field. However, it cannot be a spinor (or else the interaction term would not be a scalar) or a gaugefield (or else the interaction term would not be gauge invariant). Therefore, only a neutral scalar field remains as possible candidate. The only scalar field within the SM is the Higgs, which is an $S U(2)_{L}$ doublet. Thus, only power counting renormalizable terms have to be considered. In addition to the free Lagrangian density (20) and quartic Elko self-interactions there is a possible Elko-Higgs

\footnotetext{
4 We do not intend to discuss renormalizability which is tricky for non-local theories, but rather impose only simple power counting arguments in order to extract the dominant terms in the low energy limit.
}

interaction

$$
\left.\mathcal{L}^{\mathrm{H}}=\alpha_{H} \phi^{2}(x)\right\urcorner \bar{\eta}(x) \eta(x),
$$

where $\phi(x)$ is the Higgs doublet and $\alpha_{H}$ is a dimensionless coupling constant. The fact that Elko may not interact directly with non-abelian gauge fields ${ }^{5}$ or fermions of the SM explains why Elko has not been detected yet. However, since it does interact with the Higgs there is a chance that it might be discovered at LHC. Thus, due to its weak interaction with the matter content of the SM Elko provides a first-principle candidate for dark matter.

Conclusions. - Perhaps it not too provocative an assertion that whatever dark matter is, one thing that seems reasonably assured is that in the low-energy limit it behaves as one of the representations of the Lorentz group. Since the known particles are described by quantum fields involving finite dimensional representation spaces, and since none of them fits the properties called for by dark matter, one is guided to study the matter content of the unexplored Wigner classes. Here, we have examined one such spin one half representation space. It is emphasized that all our findings depend crucially on a single postulate: neutrality, as encoded in Eq. (4).

Not only do our results offer a possible new candidate for dark matter, but they also provide unexpected theoretical insights into the particle content of the spacetime symmetries.

\section{Acknowledgments}

We are grateful to Terry Pilling and Dima Vassilevich for helpful discussions. CONACyT (Mexico) is acknowledged for funding this research through Project 32067-E. D. Grumiller is supported by project J-2330-N08 of the Austrian Science Foundation (FWF).

Note added. - During the time this paper was under review Ref. 11] appeared. In that paper da Rocha and Rodrigues calculate the bilinear covariants for the Elko spinor fields and show that Elko belongs to class 5 in Lounesto spinor classification [12]. They further discuss distinction between Elko and Majorana spinors. In addition, if Elko is to serve as a dark matter candidate in the standard model of cosmology, Ref. [8] provides an estimate for the Elko mass (about $20 \mathrm{MeV}$ ) and the relevant cross section (roughly $2 \mathrm{pb}$ ). A refinement of that analysis in the form of an S-matrix calculation is desirable. First steps in this direction are also provided in

\footnotetext{
5 While Elko may carry a coupling to an abelian gauge field with associated field strength $F_{\mu \nu}(x)$, e.g. of the form $\vec{\eta}$ $(x) \gamma^{\mu} \gamma^{\nu} F_{\mu \nu}(x) \eta(x)$, the coupling constant has to be very small because such terms affect photon propagation. Thus, the dominant interaction between Elko and particles of the SM is expected to be via (21). We thank Dima Vassilevich for raising a question in this regard.
} 
Ref. [8], where the impact of non-locality on a perturbative treatment has been studied to a certain extent.
In particular, non-standard contractions emerge in the analogue of Wick's theorem.
[1] S. Weinberg, The Quantum Theory of Fields, vol. I, II (Cambridge University Press, 1995).

[2] D. A. Eliezer and R. P. Woodard, Nucl. Phys. B325, 389 (1989).

[3] C. Rovelli, Living Rev. Rel. 1, 1 (1998), gr-qc/9710008.

[4] M. R. Douglas and N. A. Nekrasov, Rev. Mod. Phys. 73, 977 (2001), hep-th/0106048. R. J. Szabo, Phys. Rept. 378, 207 (2003), hep-th/0109162.

[5] E. P. Wigner, Annals Math. 40, 149 (1939), E. P. Wigner, in Group theoretical concepts and methods in elementary particle physics, Lectures of the Istanbul summer school of theoretical physics (1962), edited by F. Gürsey (Gordon and Breach, New York, 1964), pp. 37-80.

[6] T. D. Lee and G. C. Wick, Phys. Rev. 148, 1385 (1966).

[7] L. H. Ryder, Quantum Field Theory (Cambridge University Press, Cambridge, 1996). D. V. Ahluwalia, Found. Phys. 28, 527 (1998), physics/9702005. F. H. Gaioli and E. T. Garcia Alvarez, Am. J. Phys. 63, 177 (1995), hep- th/9807211. See also Sec. 5.5 of vol I of [1]. It provides another important perspective on the matter. Note, in particular, the relation to parity covariance.

[8] D. V. Ahluwalia-Khalilova and D. Grumiller, J. Cosmol. Astropart. Phys. 07 (2005) 012, hep-th/0412080.

[9] D. V. Ahluwalia, M. B. Johnson, and T. Goldman, Phys. Lett. B316, 102 (1993), hep-ph/9304243.

[10] V. V. Dvoeglazov, Int. J. Theor. Phys. 34, 2467 (1995), hep-th/9504158.

[11] R. da Rocha, W. A. J. Rodrigues, math-ph/0506075 [Mod. Phys. Lett. A (to be published)].

[12] P. Lounesto, Clifford Algebras and Spinors (Cambridge University Press, Cambridge, England, 2002); see Chaps. 11 and 12 of the second edition.

[13] R. Rajaraman, Solitons and instantons: An introduction to solitons and instantons in quantum field theory (North-Holland, Amsterdam, 1982). 